\title{
Consolidação dos tubérculos na artroplastia reversa do ombro após fratura proximal do úmero: Existe melhoria nos resultados funcionais?
}

\section{Tuberosity Healing after Reverse Shoulder Arthroplasty for Proximal Humerus Fractures: Is there Clinical Improvement?}

Luís Henrique Barros ${ }^{1}$ Sérgio Figueiredo ${ }^{2}$ Manuel Marques ${ }^{3}$ Claudia Rodrigues $^{1}$ Joaquim Ramos ${ }^{1}$ Rui Claro ${ }^{1}$

1 Departamento de Ortopedia, Centro Hospitalar e Universitário do Porto, Porto, Portugal

2 Departamento de Ortopedia, Centro Hospitalar de Leiria, Leiria, Portugal

${ }^{3}$ Departamento de Ortopedia, Centro Hospitalar do Litoral Alentejano, Santiago do Cacém, Portugal

Rev Bras Ortop 2020;55(6):748-754.

\author{
Endereço para correspondência Luís Henrique Barros, MD, Centro \\ Hospitalar e Universitário do Porto, Avenida da República, 855, \\ $1^{\circ}$ direito, Vila Nova de Gaia, 4430-040, Portugal \\ (e-mail: luisbarros8@gmail.com).
}

\begin{abstract}
Resumo
Palavras-chave

- artroplastia reversa do ombro

- consolidação dos tubérculos

- fratura em idade geriátrica

- fratura do úmero proximal

Objetivo Comparar os resultados funcionais entre pacientes com fratura complexa do úmero proximal submetidos a artroplastia reversa com tubérculos consolidados e tubérculos não consolidados. O objetivo secundário foi determinar a taxa de consolidação dos tubérculos com este tipo de prótese.

Métodos Estudo de tipo coorte, retrospectivo, com coleta prospectiva de dados. No total, 28 pacientes cumpriram os critérios de inclusão: idade superior a 65 anos, prótese reversa do ombro por fratura complexa do úmero proximal ( 3 ou 4 partes, segundo Neer), e tempo de seguimento mínimo de 24 meses. Aos seis meses, todos os pacientes foram avaliados radiograficamente quanto à consolidação dos tubérculos e divididos em dois grupos: grupo com tubérculos consolidados e grupo com tubérculos não consolidados. A avaliação funcional realizou-se segundo o sistema de pontuação de Constant, da amplitude de movimento ativo, e da Escala Visual Analógica (EVA) à data da última consulta. Registaram-se todas as complicações.

Resultados A consolidação dos tubérculos ocorreu em 21 pacientes (76,3\%). Verificou-se diferenças estatisticamente significativas no sistema de pontuação de Constant $(p<0.001)$, elevação anterior $(p=0.020)$, rotação interna $(p=0.001)$ e externa $(p=0.003)$, quando se comparou o grupo dos tubérculos consolidados com o grupo dos tubérculos não consolidados. Não houve diferenças significativas na EVA entre os 2 grupos.

Conclusão A consolidação dos tubérculos traduz uma melhoria dos resultados funcionais em pacientes submetidos a artroplastia reversa do ombro como tratamento de fraturas complexas do úmero proximal em idosos.
\end{abstract}

recebido

09 de Janeiro de 2019

aceito

15 de Agosto de 2019
DOI https://doi.org/

10.1055/s-0039-3402459. ISSN 0102-3616.
Copyright $(2020$ by Sociedade Brasileira License terms de Ortopedia e Traumatologia. Published by Thieme Revinter Publicações Ltda, Rio de Janeiro, Brazil 


\begin{abstract}
Keywords

- reverse shoulder arthroplasty

- tuberosity healing

- elderly fracture

- proximal humeral fracture

Objective To compare the functional results of patients with complex proximal humerus fracture submitted to total shoulder reverse arthroplasty with and without tuberosity healing. The secondary goal was to know the tuberosity healing rate after reverse shoulder arthroplasty with our surgical technique.

Methods A retrospective, cohort type study, with a prospective database collection. In total, 28 patients fulfilled the inclusion criteria: age $\geq 65$ years, reverse shoulder arthroplasty for complex proximal humerus fracture (type-3 or -4 , according to Neer), and a minimum of 24 months of follow-up. At six months of follow-up, all of the patients were evaluated radiographically for tuberosity, and then they were divided into 2 groups: those with healed tuberosities and those with non-healed tuberosities. A clinical evaluation using the Constant score, active range of motion and the Visual Analog Scale (VAS) at the last follow-up was also performed.

Results Tuberosity healing occurred in 21 patients (76.3\%). There were statistically significant differences in the Constant scoring system $(p<0.001)$, forward elevation $(p=0.020)$, internal rotation $(p=0.001)$ and external rotation $(p=0.003)$ when comparing the group of healed tuberosities with the group of non-healed tuberosities. No differences were found regarding the VAS score.

Conclusion Tuberosity healing results in an improvement of the functional outcomes of patients submitted to reverse shoulder arthroplasty as a treatment for complex proximal humeral fractures in the elderly.
\end{abstract}

\section{Introdução}

Nos últimos 20 anos tem-se verificado um aumento da incidência das fraturas do úmero proximal (FUPs), representando $5 \%$ de todas as fraturas, sendo atualmente a terceira fratura mais frequente entre os idosos. ${ }^{1,2}$ Além disso, nesta população, as fraturas tendem a ser mais complexas, como fraturas em três ou quatro partes, e fraturas-luxações. ${ }^{3}$ Estes dados demonstram que as FUPs representam um problema de saúde pública, com tendência a agravar à medida que a população envelhece.

Na maioria das FUPs, principalmente nas mais simples, o tratamento conservador permanece o tratamento de eleição, com bons resultados. ${ }^{4,5}$ Contudo, nas fraturas em três ou quatro partes em idosos, o tratamento ideal ainda é um tópico de debate. A decisão deve ser individualizada, dependendo das comorbidades, necessidades funcionais, qualidade do osso, e experiência do cirurgião. ${ }^{6}$ Cada vez mais estudos sugerem que, nas fraturas com grande cominuição e em doentes com osso osteoporótico, a redução aberta e osteossíntese (RAO) e a hemiartroplastia (HA) não são recomendadas. ${ }^{7}$ Nestes casos, a RAO está associada a elevadas taxas de necrose avascular da cabeça umeral, perda da redução e destruição da glenoide pelos parafusos. As HAs têm demonstrando resultados pouco animadores e imprevisíveis: ${ }^{8}$ um estudo randomizado ${ }^{9}$ demonstrou resultado funcional semelhante ao do tratamento conservador. A distribuição bimodal dos resultados das HA, em excelentes ou muito ruins, depende da consolidação dos tubérculos, ${ }^{10}$ que geralmente está diminuída em pacientes com osso osteoporóticos e/ou fraturas cominutivas, ou seja, nos idosos. ${ }^{11,12}$
Assim, as próteses reversas do ombro (PROs) têm ganho popularidade no tratamento destas fraturas, pois apresentam resultados mais consistentes e previsíveis. ${ }^{8}$ Garrigues et al. ${ }^{13}$ concluíram que os pacientes com PRO apresentam resultados funcionais superiores aos pacientes com HA. Como as PROs foram concebidas para não necessitarem do manguito rotador, nos estudos iniciais não havia preocupação com a consolidação dos tubérculos. ${ }^{14}$ Contudo, tem-se verificado que a ausência do manguito rotador, principalmente do infraespinal e do redondo menor, está associada a piores resultados (diminuição da rotação externa). Alguns estudos subsequentes demonstraram que a consolidação dos tubérculos leva a resultados funcionais superiores, tanto nas PROs quanto nas HAs. ${ }^{8,15,16}$ Apesar de haver consciência da importância da consolidação dos tubérculos na obtenção de melhores resultados funcionais, existem poucos estudos que demonstrem o impacto clínico para o doente.

O principal objetivo deste trabalho foi comparar os resultados funcionais entre pacientes com FUPs complexas submetidos a PRO com tubérculos consolidados e tubérculos não consolidados. $O$ objetivo secundário foi determinar a taxa de consolidação dos tubérculos com este tipo de prótese.

\section{Material e Métodos}

\section{Desenho do Estudo}

Após aprovação pelo Conselho de Ética da instituição, foi desenvolvido um estudo tipo coorte, retrospectivo, com coleta prospectiva de dados, entre janeiro de 2011 e dezembro de 2015, no qual se pretendeu avaliar a consolidação dos 


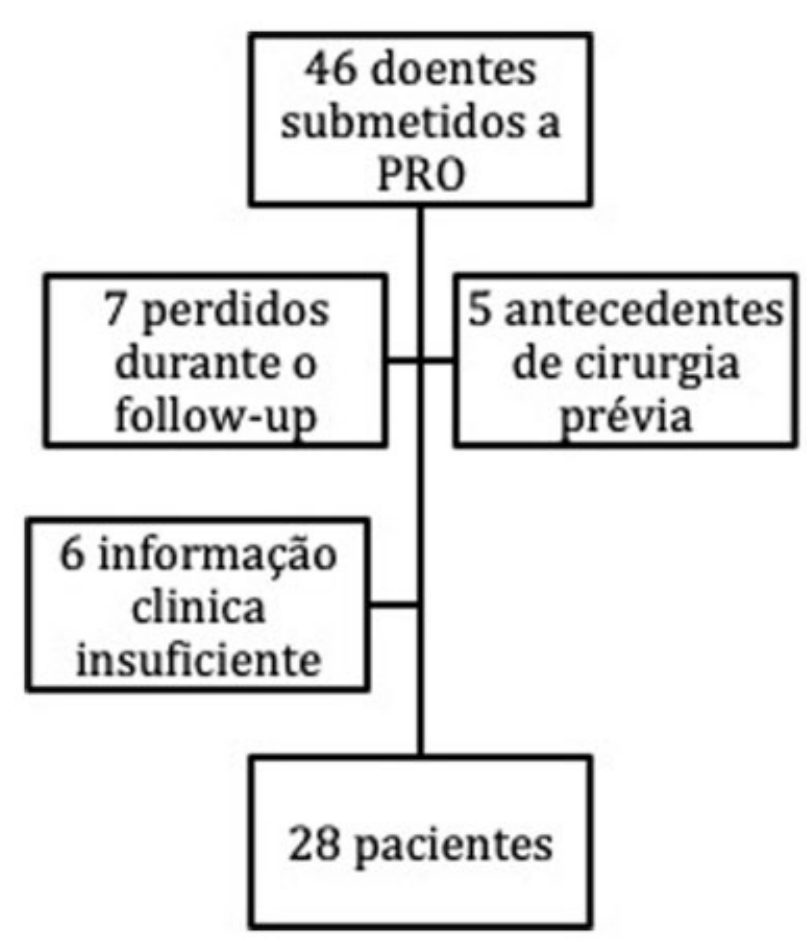

Fig. 1 Pacientes incluídos no estudo após aplicação dos critérios de inclusão e exclusão.

tubérculos em doentes idosos submetidos a PRO por FUP. Os critérios de inclusão foram: pacientes com idade $\geq 65$ anos, submetidos a PRO por FUP complexa em 3 ou 4 partes, segundo Neer, ${ }^{5}$ com ou sem luxação, e tempo de seguimento mínimo de 24 meses. Excluíram-se pacientes com cirurgia local prévia e tempo desde o evento traumático até cirurgia superior a quatro semanas. As cirurgias foram realizadas por dois ortopedistas seniores dedicados a patologias do ombro, após consentimento do paciente.

Durante o período de estudo, 46 pacientes foram submetidos a PRO por FUP. Após a aplicação dos critérios de inclusão e exclusão, 28 pacientes integraram este estudo ( - Figura $\mathbf{1}$ ).

\section{Técnica Cirúrgica}

As cirurgias foram realizadas sob anestesia geral, em posição de "cadeira de praia", por meio da via deltopeitoral. Foi utilizada sempre a mesma prótese (Aequalis-Reversed Fracture, Tornier, Edina, MN, EUA).

Intraoperatoriamente, foram mais uma vez avaliados os sinais de irreparabilidade da fratura: a) cominuição dos tubérculos; b) fragmentos indistinguíveis; c) osso osteoporótico; d) desvio acentuado da fratura com cominuição do calcar.

O tendão da longa porção do bicípite (LPB) e a sua goteira serviram de referências para a inserção dos tubérculos. Foram realizadas tenodese da LPB e excisão do tendão do supraespinal sistematicamente. A fixação dos tubérculos foi preparada nesta fase com passagem de quatro fios não absorvíveis (Ethibond no. 5, Ethicon, Somerville, Nova Jersey, USA), dois pelo tendão do infraespinal, e dois pelo redondo menor. Nas fraturas em quatro partes, dois fios adicionais foram passados pelo tendão do subescapular.
Após a preparação do canal, efetuou-se medição da altura da haste utilizando-se o calcar e a redução dos tubérculos como referências. Na ausência de calcar, foi avaliada a tensão das partes moles após a redução da prótese: tensão do músculo deltoide e tendão conjunto, assim como a distância entre o bordo superior do músculo peitoral maior e o acrômio, que deve ser de cerca de $5,6 \mathrm{~cm} .{ }^{17}$ Relativamente à versão da prótese foi usado o guia do sistema, tendo como referência o antebraço, utilizando $20^{\circ}$ de retroversão para conferir maior estabilidade. ${ }^{18}$

A glenoide foi preparada, e os componentes definitivos foram colocados. As hastes foram cimentadas distalmente à região metafisária, permitindo uma fixação hibrida. Após a secagem do cimento, efetuou-se a redução da prótese, avaliouse novamente a altura e a tensão das partes moles, e escolheuse o tamanho do polietileno. As partes moles estavam devidamente tensionadas quando a luxação após a aplicação de forças axiais e laterais era difícil de conseguir. ${ }^{19}$

A fixação dos tubérculos foi realizada de acordo com a técnica descrita por Pascal Boileau, ${ }^{20}$ utilizando os fios não absorvíveis colocados anteriormente e adicionando outros dois fios semelhantes passados através de dois furos realizados na diáfise umeral previamente à cimentação. Assim, após a redução dos tubérculos, os fios dos tendões infraespinal, redondo menor e subescapular fixam os tubérculos como cerclages horizontais, e os fios que passam na diáfise neutralizam a construção como duas bandas de tensão verticais (uma para cada tubérculo).

No pós-operatório, os pacientes mantiveram o membro imobilizado com suporte por três semanas, começando então movimentos pendulares. Movimentos de elevação e abdução só foram permitidos após seis semanas, iniciando de seguida reabilitação fisiátrica com o devido acompanhamento.

\section{Avaliação Radiológica}

A consolidação dos tubérculos foi avaliada radiograficamente utilizando as incidências em rotação neutra, interna e externa, e de perfil aos seis meses após a cirurgia. ${ }^{11}$ Quando os tubérculos se encontravam acima do polietileno, estavam a uma distância superior a $1 \mathrm{~cm}$ da sua posição anatómica, ou não estavam presentes, considerou-se não haver consolidação dos tubérculos. Sempre que alguma dúvida subsistisse, foi pedida tomografia computorizada (TC) aos seis meses após a cirurgia como ferramenta adicional, o que permitiu avaliar a existência de continuidade óssea entre a diáfise e os tubérculos. A classificação das fraturas e a consolidação dos tubérculos foram determinadas por dois cirurgiões especialistas em cirurgia de ombro, e sempre que houvesse divergência de respostas foi pedida opinião a um terceiro cirurgião especialista em cirurgia de ombro.

\section{Avaliação Funcional}

Todos os pacientes foram avaliados com 2, 6 e 12 semanas, com 6 e 12 meses, e anualmente. Os resultados funcionais foram determinados por meio do sistema de pontuação de Constant (Constant Score, CS), do registo da amplitude de movimento ativo em elevação anterior (EA), abdução, rotação externa (RE) e interna (RI). A amplitude de movimento foi medida em graus 


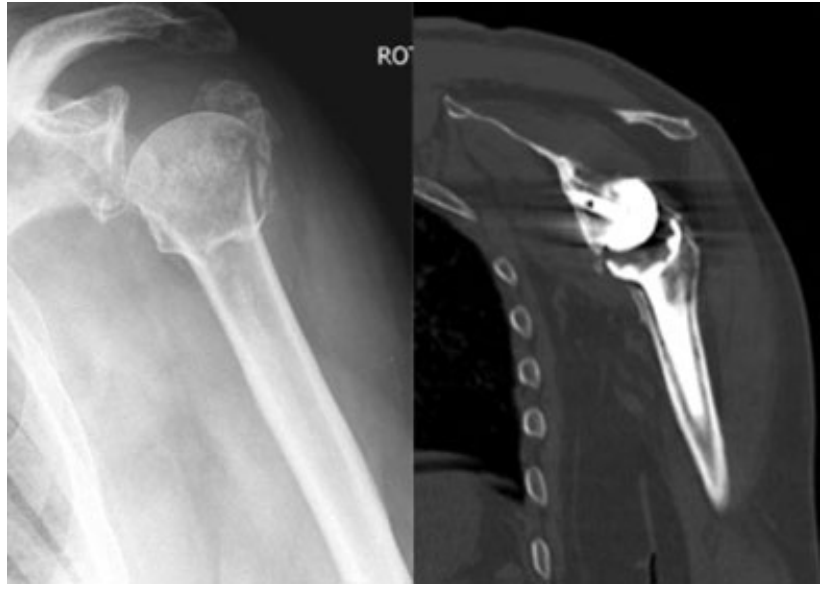

Fig. 2 Tomografia computorizada demonstrando tubérculos consolidados.

com goniômetro, e as rotações, com o cotovelo junto ao corpo. Foi também avaliada a Escala Visual Analógica (EVA), e foram registadas as complicações intra e pós-operatórias. Os resultados dos pacientes com tubérculos consolidados e tubérculos não consolidados foram comparados.

\section{Análise Estatística}

Foram comparadas as médias do CS, da amplitude de movimento, e da VAS por teste não paramétrico U de MannWhitney, dada a dimensão amostral. Para as variáveis binomiais, foi usado o teste do qui-quadrado de Pearson, salvaguardando contagem em células igual ou superior a 5 .

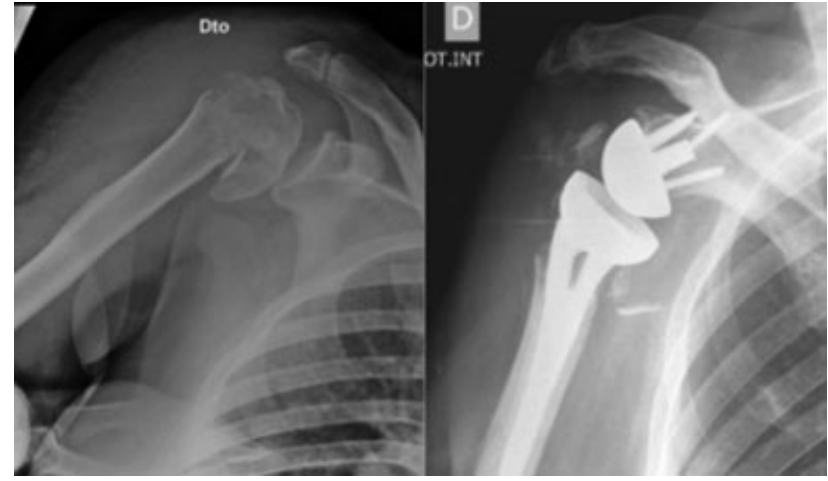

Fig. 3 Radiografia demonstrando tubérculos não consolidados.

\section{Resultados}

\section{Dados Epidemiológicos}

Foram incluídos 28 pacientes, 22 (78.6\%) do gênero feminino, e $6(21.4 \%)$ do gênero masculino, com idade média de 70,1 anos (mínima: 65,2 anos; máxima: 89,8 anos). Obteve-se 5 fraturas em 3 partes e 23 em 4 partes. 0 tempo de seguimento médio foi de 58,4 meses (32,5 a 88,2 meses).

\section{Consolidação dos Tubérculos}

A consolidação ocorreu em 21 (75\%) pacientes (-Figura 2). No total, 3 pacientes apresentaram reabsorção dos tubérculos, e 4 tiveram migração dos tubérculos, representando um total de 7 (25\%) pacientes com tubérculos não consolidados (-Figura 3).

Tabela 1 Consolidação dos tubérculos e distribuição de variáveis independentes pelos dois grupos

\begin{tabular}{|l|l|l|l|}
\hline \multirow{2}{*}{ Variável } & Consolidação de tubérculos & \multirow{2}{*}{ Valor de $\boldsymbol{p}$} \\
\cline { 2 - 3 } & $\begin{array}{l}\text { Sim } \\
(\boldsymbol{n}=\mathbf{2 1})\end{array}$ & $\begin{array}{l}\text { Não } \\
(\boldsymbol{n}=\mathbf{7})\end{array}$ & \\
\hline Sexo feminino (\%) & $16(76,2 \%)$ & $6(85,7 \%)$ & $0,595^{*}$ \\
\hline $\begin{array}{l}\text { Idade mediana } \\
\text { [mínima - máxima] }\end{array}$ & $73,6[60,2-82,1]$ & $70,9[60,7-89,8]$ & $0,876^{* *}$ \\
\hline Seguimento mediano (meses) [mínimo - máximo] & $56,5[33,2-88,2]$ & $46,8[32,5-73,1]$ & $0,140^{* *}$ \\
\hline
\end{tabular}

Notas: distribuição de variáveis independentes por grupos de estudo e respectiva comparação estatística, não se observando diferenças estatisticamente significativas para qualquer uma. ${ }^{*}$ Teste do qui-quadrado. ${ }^{* *}$ Teste $U$ de Mann-Whitney.

Tabela 2 Comparação dos resultados funcionais entre os dois grupos (consolidação dos tubérculos e não consolidação dos tubérculos)

\begin{tabular}{|l|l|l|l|}
\hline \multirow{2}{*}{ Avaliação funcional } & Consolidação dos tubérculos & \multirow{2}{*}{ Valor de $\boldsymbol{p}$} \\
\cline { 2 - 4 } & $\begin{array}{l}\text { Sim } \\
(\boldsymbol{n}=\mathbf{2 1})\end{array}$ & $\begin{array}{l}\text { Não } \\
(\boldsymbol{n}=7)\end{array}$ & \\
\hline Pontuação de Constant & $79,0(5 ; 0)$ & $55,0(11 ; 0)$ & $p<0,001^{*}$ \\
\hline Elevação anterior & $135,0^{\circ}\left(46 ; 0^{\circ}\right)$ & $90,0^{\circ}\left(70 ; 0^{\circ}\right)$ & $p=0,02^{*}$ \\
\hline Abdução & $105,0^{\circ}\left(45 ; 0^{\circ}\right)$ & $75,0^{\circ}\left(60 ; 0^{\circ}\right)$ & $p=0,435$ \\
\hline Rotação interna & $60,0^{\circ}\left(45 ; 0^{\circ}\right)$ & $30,0^{\circ}\left(0 ; 0^{\circ}\right)$ & $p=0,01^{*}$ \\
\hline Rotação externa & $60,0^{\circ}\left(32 ; 5^{\circ}\right)$ & $30,0^{\circ}\left(0 ; 0^{\circ}\right)$ & $p=0,03^{*}$ \\
\hline Escala Visual Analógica & $1,0(0 ; 5)$ & $1,0(1 ; 0)$ & $p=0,836$ \\
\hline
\end{tabular}

Notas: os valores das variáveis contínuas foram apresentados como a mediana e o respectivo valor de percentil 25 e percentil 75 . ${ }^{*} p<0,05$. 


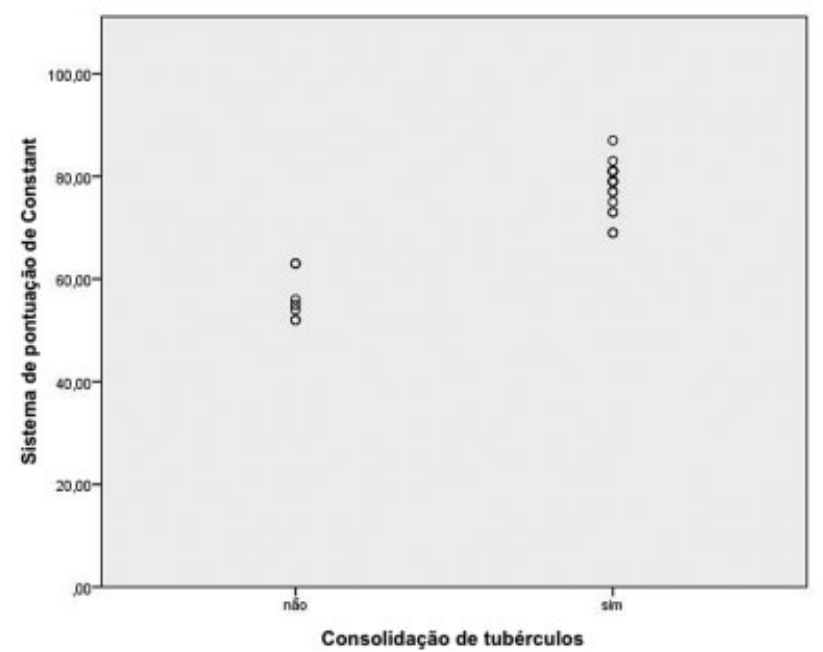

Fig. 4 Distribuição da pontuação de Constant por grupos (consolidação versus não consolidação).

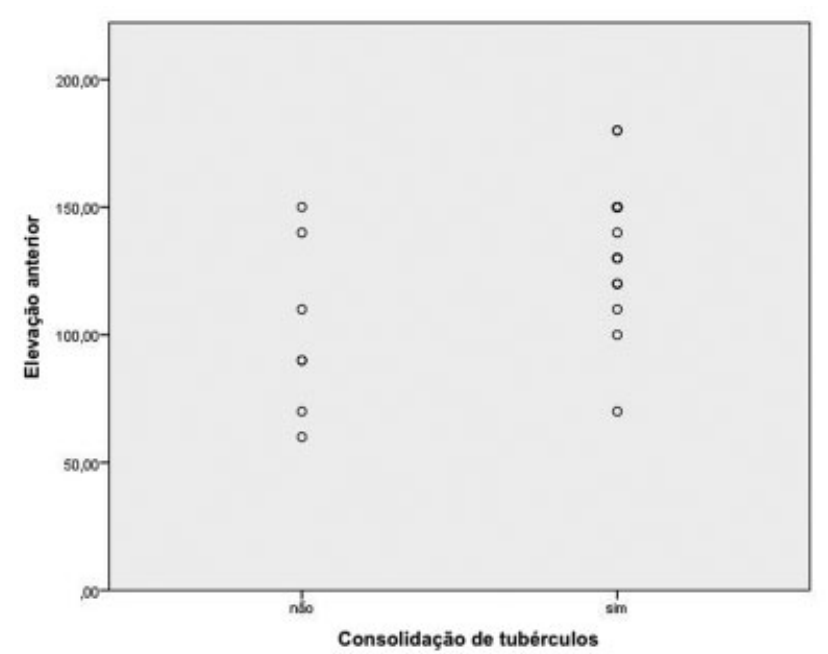

Fig. 5 Distribuição da elevação anterior do ombro por grupos (consolidação versus não consolidação).

A - Tabela 1 apresenta a distribuição das variáveis independentes pelos dois grupos.

\section{Resultados Funcionais}

A maioria dos pacientes (75.0\%) estavam sem dor em repouso e durante as suas atividades diárias.

As diferenças nos resultados funcionais entre pacientes com tubérculos consolidados e não consolidados estão descritas na - Tabela 2. Obtiveram-se diferenças estatisticamente significativas no CS, EA, RI e RE. As - Figuras 4-8 demonstram a distribuição das variáveis em estudo por esses 2 grupos.

Na análise multivariada da influência potencial do gênero e/ou da idade nos resultados obtidos, constatou-se a não interferência com relevância estatística com relação ao CS $(p=0.630$ e $p=0.868$, respetivamente), EA $(p=0.157$ e $p=0.853$, respetivamente), abdução ( $p=0.566$ e $p=0.497$, respetivamente), RI ( $p=0.431$ e $p=0.601$, respetivamente),

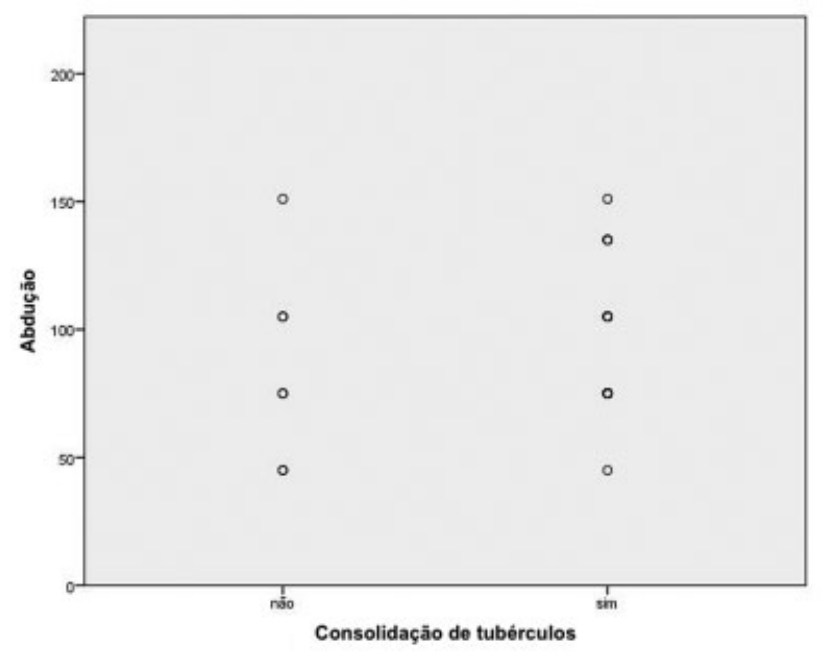

Fig. 6 Distribuição da abdução do ombro por grupos (consolidação versus não consolidação).

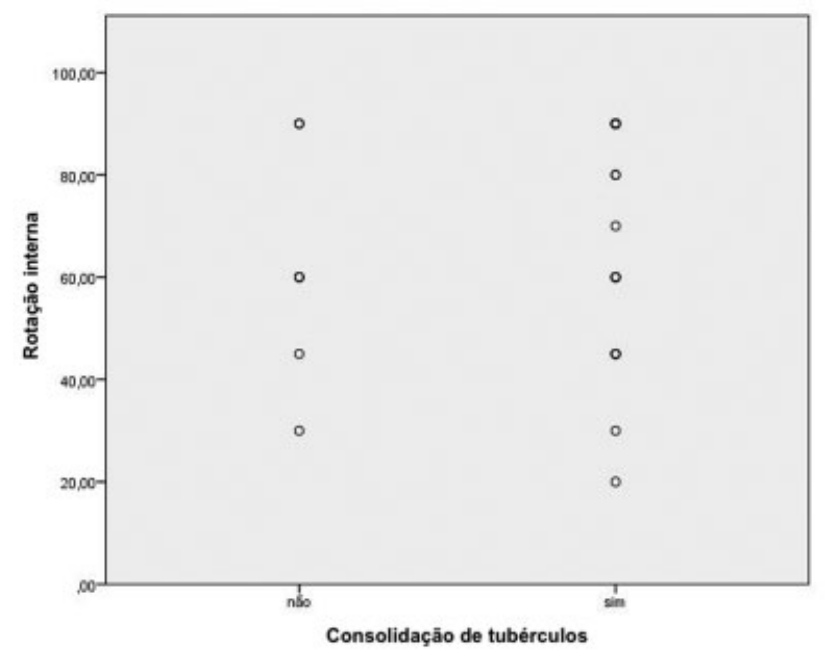

Fig. 7 Distribuição da rotação interna do ombro por grupos (consolidação versus não consolidação).

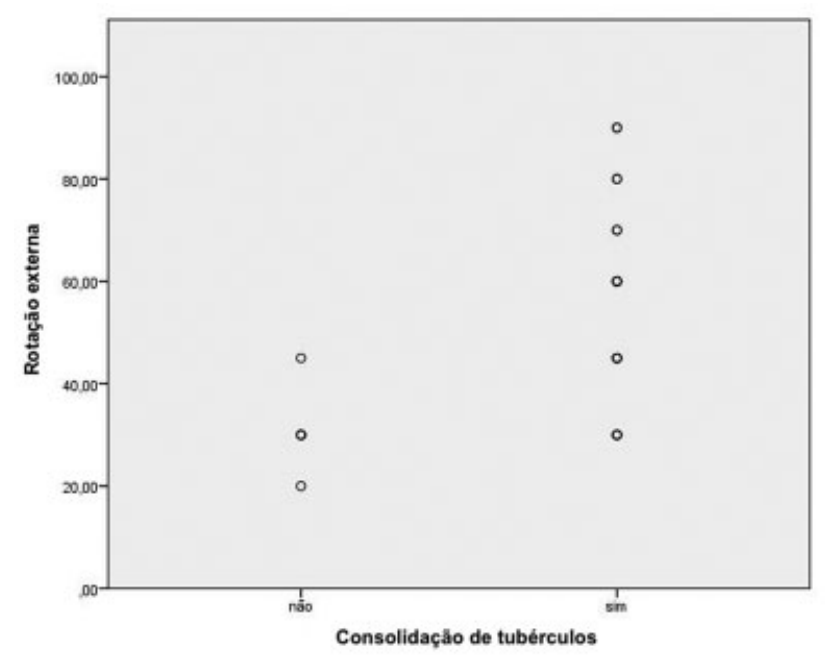

Fig. 8 Distribuição da rotação externa do ombro por grupos (consolidação versus não consolidação). 
$\operatorname{RE}(p=0.239$ e $p=0.526$, respetivamente $)$, e VAS $(p=0.164 \mathrm{e}$ $p=0.722$, respetivamente). Contudo, isolando a consolidação como variável no modelo, apenas o CS $(p<0.001)$ e a RI $(p=0.002) \quad$ mantiveram relação estatisticamente significativa.

\section{Complicações}

Não houve nenhum episódio de luxação ou instabilidade.

Verificou-se uma infeção superficial aguda, submetida a desbridamento, correspondendo a um dos casos de não consolidação dos tubérculos.

\section{Discussão}

O uso de PRO em pacientes com FUP está em crescimento, principalmente em idosos. Inicialmente, a longevidade destas próteses causava preocupação, mas os bons resultados verificados em estudos em médio e longo prazos tem ampliado o uso delas. Du et al. ${ }^{21}$ verificaram que as PROs estavam associadas a um aumento significativo do CS e diminuição de reintervenções, quando comparado com as HAs. Klein et al. ${ }^{16}$ também relataram melhoria no CS e um formulário curto de pesquisa em saúde. Em estudo prospectivo realizado por Bufquin et al., ${ }^{15}$ em que foram avaliados pacientes com FUPs complexas submetidos a PRO, foi concluído que a não consolidação dos tubérculos não implica necessariamente um mau resultado final. Torrens et al. $^{22}$ afirmam que os resultados clínicos são sobreponíveis, não dependendo da consolidação dos tubérculos. As PROs se baseiam nos princípios clássicos da prótese desenvolvida por Grammont: a) uma prótese semiconstritiva com ponto de fixação (fulcro) fixo, a partir do qual o deltoide realiza elevação do braço sem necessidade de recurso dos manguitos rotadores, e b) centro de rotação medializado e mais baixo, o que permite alongar o deltoide e assim diminuir a força necessária para realizar abdução do braço. $^{23,24}$ Deste modo, percebe-se que as PROs podem demonstrar bons resultados mesmo sem a consolidação dos tubérculos. Apesar disto, estudos mais recentes sugerem que a integração dos tubérculos e o bom funcionamento dos manguitos rotadores apresentam um benefício funcional para os pacientes, com aumento da amplitude de movimento, particularmente das rotações. No nosso estudo, verificamos uma melhoria estatisticamente significativa na EA ( $127.5^{\circ}$ versus 101.4$)$, na $\mathrm{RE}\left(60^{\circ}\right.$ versus $\left.30^{\circ}\right)$, e na $\mathrm{RI}$ $\left(64.8^{\circ}\right.$ versus $\left.34.3^{\circ}\right)$. Estes resultados reforçam os dados demonstrados encontrados na literatura. Lenarz et al. ${ }^{25}$ descreveram uma EA de $139^{\circ}$, e uma RE de $27^{\circ}$, e Valenti et al. ${ }^{26}$ relataram CS de 55 , EA de $112^{\circ}$, abdução de $97^{\circ}$, e RE de $12.7^{\circ}$. Gallinet et al. ${ }^{8}$ demonstraram EA de $104^{\circ}$ e uma RE de $33^{\circ}$. Uma revisão sistemática recente resume bem os resultados destes estudos, concluindo que a consolidação dos tubérculos parece traduzir-se em resultados funcionais superiores, principalmente nas rotações. ${ }^{27}$ Além disso, uma pequena melhoria na amplitude de movimento dos pacientes pode traduzir-se numa melhoria real na sua qualidade de vida. No nosso trabalho, verificamos que o aumento da mobilidade dos pacientes correspondeu à melhoria do CS
(79 versus 55). Boileau et al. ${ }^{28}$ resumem seu estudo afirmando não só que a consolidação dos tubérculos melhora a EA e a RE como também, a qualidade de vida dos doentes.

Com relação ao segundo objetivo do trabalho, verificamos consolidação dos tubérculos em $76.3 \%$ dos pacientes. Esta percentagem está de acordo com estudos mais recentes, ${ }^{22,29,30}$ e é superior a trabalhos mais antigos. Boileau et al. $^{28}$ descrevem uma taxa de consolidação superior, de $84 \%$, defendendo que a consolidação dos tubérculos nas PROs por fratura é um procedimento reprodutível mesmo em idosos. Também as próteses evoluíram, e a existência de próteses desenhadas especificamente para fraturas, com janela na região metafisária, permitem melhor integração e consolidação dos tubérculos.

Alguns estudos defendem a melhoria em todos os planos com a consolidação dos tubérculos. ${ }^{29}$ Em nosso estudo, não tivemos melhoria significativa na abdução $\left(106^{\circ}\right.$ versus $\left.83^{\circ}\right)$, mas esta foi superior nos pacientes com tubérculos consolidados. A EVA foi semelhante nos dois grupos, observandose que a consolidação dos tubérculos não interfere na dor.

O procedimento demonstrou baixa incidência de complicações, com apenas uma infecção, completamente debelada com desbridamento e antibioterapia. Não se verificaram luxações, instabilidades ou lesões neurológicas.

As limitações deste trabalho são aquelas inerentes aos estudos retrospectivos. Além disso, o uso de testes não paramétricos, devido ao fato de a amostra ser relativamente pequena, não permite ampla discriminação na análise dos dados, apesar de os resultados obtidos serem estatisticamente significativos. São necessários mais estudos randomizados para comprovar definitivamente a superioridade dos resultados funcionais com a consolidação dos tubérculos.

Concluindo, nosso trabalho demonstrou superioridade no CS, EA, RE e RI quando a consolidação dos tubérculos foi conseguida, o que correspondeu a 78.6\% dos casos.

Conflito de Interesses

Os autores declaram não haver conflito de interesses.

\section{Referências}

1 Sanders RJ, Thissen LG, Teepen JC, van Kampen A, Jaarsma RL. Locking plate versus nonsurgical treatment for proximal humeral fractures: better midterm outcome with nonsurgical treatment. J Shoulder Elbow Surg 2011;20(07):1118-1124

2 Khatib O, Onyekwelu I, Zuckerman JD. The incidence of proximal humeral fractures in New York State from 1990 through 2010 with an emphasis on operative management in patients aged 65 years or older. J Shoulder Elbow Surg 2014;23(09):1356-1362

3 Court-Brown CM, Garg A, McQueen MM. The epidemiology of proximal humeral fractures. Acta Orthop Scand 2001;72(04): 365-371

4 Court-Brown CM, Garg A, McQueen MM. The translated two-part fracture of the proximal humerus. Epidemiology and outcome in the older patient. J Bone Joint Surg Br 2001;83(06):799-804

5 Neer CS II. Displaced proximal humeral fractures. I. Classification and evaluation. J Bone Joint Surg Am 1970;52(06):1077-1089

6 Maier D, Jaeger M, Izadpanah K, Strohm PC, Suedkamp NP. Proximal humeral fracture treatment in adults. J Bone Joint Surg Am 2014;96(03):251-261 
7 Jobin CM, Galdi B, Anakwenze OA, Ahmad CS, Levine WN. Reverse shoulder arthroplasty for the management of proximal humerus fractures. J Am Acad Orthop Surg 2015;23(03):190-201

8 Gallinet D, Clappaz P, Garbuio P, Tropet Y, Obert L. Three or four parts complex proximal humerus fractures: hemiarthroplasty versus reverse prosthesis: a comparative study of 40 cases. Orthop Traumatol Surg Res 2009;95(01):48-55

9 Boons HW, Goosen JH, van Grinsven S, van Susante JL, van Loon CJ. Hemiarthroplasty for humeral four-part fractures for patients 65 years and older: a randomized controlled trial. Clin Orthop Relat Res 2012;470(12):3483-3491

10 Sirveaux F, Roche O, Molé D. Shoulder arthroplasty for acute proximal humerus fracture. Orthop Traumatol Surg Res 2010;96 (06):683-694

11 Boileau P, Winter M, Cikes A, et al. Can surgeons predict what makes a good hemiarthroplasty for fracture? J Shoulder Elbow Surg 2013;22(11):1495-1506

12 Boileau P, Krishnan SG, Tinsi L, Walch G, Coste JS, Molé D. Tuberosity malposition and migration: reasons for poor outcomes after hemiarthroplasty for displaced fractures of the proximal humerus. J Shoulder Elbow Surg 2002;11(05):401-412

13 Garrigues GE, Johnston PS, Pepe MD, Tucker BS, Ramsey ML, Austin LS. Hemiarthroplasty versus reverse total shoulder arthroplasty for acute proximal humerus fractures in elderly patients. Orthopedics 2012;35(05):e703-e708

14 Cazeneuve JF, Cristofari DJ. The reverse shoulder prosthesis in the treatment of fractures of the proximal humerus in the elderly. J Bone Joint Surg Br 2010;92(04):535-539

15 Bufquin T, Hersan A, Hubert L, Massin P. Reverse shoulder arthroplasty for the treatment of three- and four-part fractures of the proximal humerus in the elderly: a prospective review of 43 cases with a short-term follow-up. J Bone Joint Surg Br 2007;89 (04):516-520

16 Klein M, Juschka M, Hinkenjann B, Scherger B, Ostermann PA. Treatment of comminuted fractures of the proximal humerus in elderly patients with the Delta III reverse shoulder prosthesis. J Orthop Trauma 2008;22(10):698-704

17 Murachovsky J, Ikemoto RY, Nascimento LG, Fujiki EN, Milani C, Warner JJ. Pectoralis major tendon reference (PMT): a new method for accurate restoration of humeral length with hemiarthroplasty for fracture. J Shoulder Elbow Surg 2006;15 (06):675-678

18 Nalbone L, Adelfio R, D'Arienzo M, et al. Optimal positioning of the humeral component in the reverse shoulder prosthesis. Musculoskelet Surg 2014;98(02):135-142
19 Lädermann A, Edwards TB, Walch G. Arm lengthening after reverse shoulder arthroplasty: a review. Int Orthop 2014;38 (05):991-1000

20 Pascal Boileau GW, Sumant G. Krishnan. Tuberosity Osteosynthesis and Hemiarthroplasty for Four-Part Fractures of the Proximal Humerus. Tecnhiques in Shoulder and Elbow Surgery 2000

21 Du S, Ye J, Chen H, Li X, Lin Q. Interventions for Treating 3- or 4part proximal humeral fractures in elderly patient: A network meta-analysis of randomized controlled trials. Int J Surg 2017; 48:240-246

22 Torrens C, Alentorn-Geli E, Mingo F, Gamba C, Santana F. Reverse shoulder arthroplasty for the treatment of acute complex proximal humeral fractures: Influence of greater tuberosity healing on the functional outcomes. J Orthop Surg (Hong Kong) 2018;26(01): 2309499018760132

23 Henninger HB, Barg A, Anderson AE, Bachus KN, Tashjian RZ, Burks RT. Effect of deltoid tension and humeral version in reverse total shoulder arthroplasty: a biomechanical study. J Shoulder Elbow Surg 2012;21(04):483-490

24 Boileau P, Watkinson DJ, Hatzidakis AM, Balg F. Grammont reverse prosthesis: design, rationale, and biomechanics. J Shoulder Elbow Surg 2005;14(1, Suppl S)147S-161S

25 Lenarz C, Shishani Y, McCrum C, Nowinski RJ, Edwards TB, Gobezie R. Is reverse shoulder arthroplasty appropriate for the treatment of fractures in the older patient? Early observations. Clin Orthop Relat Res 2011;469(12):3324-3331

26 Valenti P, Katz D, Kilinc A, Elkholti K, Gasiunas V. Mid-term outcome of reverse shoulder prostheses in complex proximal humeral fractures. Acta Orthop Belg 2012;78(04):442-449

27 Jain NP, Mannan SS, Dharmarajan R, Rangan A. Tuberosity healing after reverse shoulder arthroplasty for complex proximal humeral fractures in elderly patients-does it improve outcomes? A systematic review and meta-analysis. J Shoulder Elbow Surg 2019;28(03):e78-e91

28 Boileau P, Alta TD, Decroocq L, et al. Reverse shoulder arthroplasty for acute fractures in the elderly: is it worth reattaching the tuberosities? J Shoulder Elbow Surg 2019;28(03):437-444

29 Garofalo R, Flanagin B, Castagna A, Lo EY, Krishnan SG. Reverse shoulder arthroplasty for proximal humerus fracture using a dedicated stem: radiological outcomes at a minimum 2 years of follow-up-case series. J Orthop Surg Res 2015;10:129

30 Formaini NT, Everding NG, Levy JC, Rosas S. Tuberosity healing after reverse shoulder arthroplasty for acute proximal humerus fractures: the "black and tan" technique. J Shoulder Elbow Surg 2015;24(11):e299-e306 